\title{
A SHORT ANALYTIC PROOF OF CLOSEDNESS OF LOGARITHMIC FORMS
}

\author{
Dedicated to Professor Nobuyuki Suita on the occasion \\ of his 60 th birthday
}

JUNJIRO NOGUCHI

\section{§1. Introduction}

Deligne [D, (3.2.14)] proved the $d$-closedness of logarithmic forms on a smooth complex quasi-projective variety by showing the degeneracy of some spectral sequence. Actually, his proof works for a Zariski open subspace of a compact Kähler manifold. The logarithmic forms play important roles in various aspects of analytic-algebraic geometry, including the value distribution of holomorphic mappings (see, e.g., [D], [I], [N1], [N2], [N3] and [N4]), and their closedness is fundamental. Therefore it may be of worth, and hence our purpose of this note to give its short proof based only on the classical harmonic integral theory $[\mathrm{K}]$.

Let $D$ be an effective reduced divisor on a compact Kähler manifold $M$, and $\Omega_{M}^{p}(\log D)$ the sheaf of germs of logarithmic $p$-forms along $D$ over $M$ (see $\S 2$ for the definition).

THEOREM. Let $\omega \in H^{0}\left(M, \Omega_{M}^{p}(\log D)\right)$ be a global section of $\Omega_{M}^{p}(\log D)$. Then $d \omega=0$ in the complement $M \backslash D$ of $D$.

\section{§. Definitions and Lemmas}

In the present note we denote by $M$ an $m$-dimensional compact Kähler manifold with structure sheaf $\mathcal{O}_{M}$ of germs of holomorphic functions. Let $\Omega_{M}^{p}$ denote the sheaf of germs of holomorphic $p$-forms over $M$. Let $D$ be an effective reduced divisor on $M$. Let $x \in M$ and take irreducible $\sigma_{j} \in \mathcal{O}_{M}, x, 1 \leqq j \leqq k$, so that $\left\{\sigma_{\jmath}=0\right\}$ define the local irreducible components of $D$ at $x$. Then we define the sheaf $\Omega_{M}^{1}(\log D)$ of germs of logarithmic 1 forms along $D$ by

$$
\Omega_{M, x}^{1}(\log D)=\sum_{j=1}^{k} \mathcal{O}_{M, x} \frac{d \sigma_{\jmath}}{\sigma_{\jmath}}+\Omega_{M, x}^{1} .
$$

Received June 6, 1994. 
Moreover, we set

$$
\Omega_{M, x}^{p}(\log D)=\Omega_{M, x}^{1}(\log D) \wedge \cdots \wedge \Omega_{M, x}^{1}(\log D) \quad(p \text {-times }) .
$$

Let $\pi: \tilde{M} \rightarrow M$ be a desingularization of $D$ by Hironaka such that $\tilde{M}$ is Kähler and the divisor $\tilde{D}=\pi^{-1}(D)$ has only simple normal crossings. Then it easily follows from the extension of holomorphic functions over analytic subsets of codimension $\geqq 2$ that the pull-back

$$
\pi^{*}: H^{0}\left(M, \Omega_{M}^{p}(\log D)\right) \longrightarrow H^{0}\left(\tilde{M}, \Omega_{\widetilde{M}}^{p}(\log \tilde{D})\right)
$$

is an isomorphism. Henceforth we may assume that

$D$ has only simple normal croossings.

Let $D=\sum_{\imath=1}^{l} D_{\imath}$ be the decomposition into irreducible components, and $\iota_{D_{i}}: D_{i} \rightarrow M$ be the inclusions. Take $x \in D_{i}$. Then there is a holomorphic local coordinate system $\left(x^{1}, \cdots, x^{m}\right)$ in a neighborhood $U$ of $x$ such that $\left\{x^{1}=0\right\}=$ $D_{i} \cap U$. For $\omega \in H^{0}\left(M, \Omega_{M}^{p}(\log D)\right)$ we write

$$
\omega=\frac{d x^{1}}{x^{1}} \wedge \eta+\omega^{\prime} \quad \text { in } U
$$

so that $\eta \in H^{0}\left(U, \Omega_{M}^{p-1}\left(\log \sum_{\jmath \neq \imath} D_{\jmath}\right)\right)$ and $\omega^{\prime} \in H^{0}\left(U, \Omega_{M}^{P}\right)$ do not contain $d x^{1}$. Put

$$
\operatorname{Res}_{D_{i}}(\omega)=\ell_{D_{i}}^{*}(\eta) \quad \text { in } D_{i} \cap U \text {. }
$$

Then $\operatorname{Res}_{D_{i}}(\omega)$ is well-defined globally, and then

$$
\operatorname{Res}_{D_{i}}(\omega) \in H^{0}\left(D_{i}, \Omega_{D_{i}}^{p-1}\left(\log \sum_{j \neq \imath} D_{j} \cap D_{\imath}\right)\right) .
$$

We also consider $\operatorname{Res}_{D_{i}}(\boldsymbol{\omega})$ as a current of type $(p, 1)$ on $M$, and set

$$
\operatorname{Res}(\omega)=\sum_{i} \operatorname{Res}_{D_{i}}(\omega),
$$

which is called the Poincaré residue. We denote by $[\omega]$ the current of type $(p, 0)$ defined by $\omega$. By Stokes's theorem we have

$$
\operatorname{Res}(\omega)=\frac{1}{2 \pi i} \bar{\partial}[\omega] \text { (in the sense of currents). }
$$

Take $j \neq i$. If $D_{j} \cap D_{i}=\emptyset$, then we define

$$
\operatorname{Res}_{D_{j} \cdot D_{i}}(\omega)=\operatorname{Res}_{D_{j}}\left(\operatorname{Res}_{D_{i}}(\omega)\right)=0 .
$$

Otherwise, we define

$$
\operatorname{Res}_{D_{j} \cdot D_{i}}(\omega)=\operatorname{Res}_{D_{j} \cap D_{i}}\left(\operatorname{Res}_{D_{i}}(\omega)\right) .
$$

Let $x \in D_{j} \cap D_{i}$ and take a holomorphic local coordinate system $\left(x^{1}, \cdots, x^{m}\right)$ such that $\left\{x^{1}=0\right\}=D_{i}$ and $\left\{x^{2}=0\right\}=D_{\jmath}$, locally. Then we write 


$$
\omega=\frac{d x^{1}}{x^{1}} \wedge \eta_{1}+\frac{d x^{2}}{x^{2}} \wedge \eta_{2}+\frac{d x^{1}}{x^{1}} \wedge \frac{d x^{2}}{x^{2}} \wedge \eta_{3},
$$

where $\eta_{\nu}, 1 \leqq \nu \leqq 3$, do not contain $d x^{1}$ nor $d x^{2}$. Then

Thus we have

$$
\operatorname{Res}_{D_{j} \cdot D_{i}}(\omega)=\iota_{D_{j} \cap D_{i}}^{*} \eta_{3} \quad \text { around } x .
$$

$$
\left\{\begin{array}{l}
\operatorname{Res}_{D_{j} \cdot D_{i}}(\omega) \in H^{0}\left(D_{j} \cap D_{i}, \Omega_{D_{j} \cap D_{i}}^{p-2}\left(\log \sum_{k \neq \jmath, \imath} D_{k} \cap D_{j} \cap D_{i}\right)\right), \\
\operatorname{Res}_{D_{j} \cdot D_{i}}(\omega)+\operatorname{Res}_{D_{i} \cdot D_{j}}(\omega)=0 .
\end{array}\right.
$$

We recall a theorem from the theory of harmonic integrals. We fix a Kähler metric on $M$. As usual, we denote by $H$ the harmonic projection, by $G$ the Green operator, by $\delta$ (resp. $\vartheta, \bar{\vartheta}$ ), the adjoint of $d$ (resp. $\partial, \bar{\partial}$ ), and by $\Lambda$ the adjoint of the multiplication operator by the Kähler form (see [W]).

(2.4) Lemma ([K, Theorem 1.1.1]). Let $T$ be a current of type $(p, q+1)$ on $M$ such that $H T=d T=0$. Then the current

$$
\Theta=\frac{1}{i}(d \Lambda+i \delta) G T
$$

is of type $(p, q)$ and satisfies $d \Theta=T$, so that $d \Theta=0$ in $M \backslash \operatorname{supp} T$, where supp $T$ denotes the support of $T$.

\section{§3. Proof of Theorem}

We prove Theorem by induction on $p$, where $M$ may be arbitrary. We keep the notation in $\S 2$.

(i) The case of $p=1$. Put $T=\bar{\partial}[\omega]$. Then by (2.2) and (2.1) we have

$$
T=2 \pi i \operatorname{Res}(\boldsymbol{\omega})=\sum_{\imath} a_{\imath} D_{\imath}, \quad a_{i} \in \boldsymbol{C}^{\imath}
$$

Therefore, $H T=d T=0$ and $\operatorname{supp} T \subset D$. It follows from Lemma (2.4) that

$$
\Theta=\frac{1}{i}(d \Lambda+i \delta) G T
$$

is of type $(1,0)$ and satisfies

$$
d \Theta=T, \quad \text { so that } d \Theta=0 \text { in } M \backslash D .
$$

We put

$$
S=[\omega]-\Theta,
$$

and claim that $S$ is harmonic; i.e., $\Delta S=0$, where $\Delta$ stands for the Laplace operator. Take an arbitrary differential form $\alpha$ of type $(m-1, m)$ on $M$. Then we have 


$$
\begin{aligned}
\langle\Delta S, \alpha\rangle & =\int_{M} S \wedge \Delta \alpha=2 \int_{M} S \wedge(\bar{\partial} \bar{\vartheta} \alpha+\bar{\vartheta} \bar{\partial} \alpha) \\
& =2 \int_{M} S \wedge \bar{\partial} \bar{\vartheta} \alpha=2\left(\int_{M} \omega \wedge \bar{\partial} \bar{\vartheta} \alpha-\langle\Theta, \bar{\partial} \bar{\vartheta} \alpha\rangle\right) \\
& =2\left(\int_{M} \omega \wedge \bar{\partial} \bar{\vartheta} \alpha-\langle\Theta, d \bar{\vartheta} \alpha\rangle\right)=2(-\langle T, \bar{\vartheta} \alpha\rangle+\langle d \Theta, \bar{\vartheta} \alpha\rangle) \\
& =-2\langle T-d \Theta, \bar{\vartheta} \alpha\rangle=0 \quad \text { by (3.1). }
\end{aligned}
$$

Thus $S$ is smooth everywhere and $d S=0$. Combining this with (3.1), we deduce that $d \omega=0$ in $M \backslash D$.

(ii) Assume that Theorem holds for $p-1(p \geqq 2)$ for an arbitrary compact Kähler manifold. Take $\omega \in H^{0}\left(M, \Omega_{M}^{p}(\log D)\right)$, and put

$$
T=\grave{\partial}[\omega] .
$$

To use Lemma (2.4), we show that

$$
H T=d T=0 .
$$

The first one is trivial. We show the second. Let $\alpha$ be an arbitrary $(2 n-p-1)$ form on $M$. Then

$$
\begin{aligned}
\langle d T, \alpha\rangle & =(-1)^{p}\langle T, d \alpha\rangle \\
& =(-1)^{p} 2 \pi i\langle\operatorname{Res}(\omega), d \alpha\rangle \\
& =2 \pi i(-1)^{p} \sum_{i} \int_{D_{i}} \operatorname{Res}_{D_{i}}(\omega) \wedge d \iota_{D_{i}}^{*} \alpha .
\end{aligned}
$$

It follows from (2.1) and the induction hypothesis that

$$
d \operatorname{Res}_{D_{i}}(\boldsymbol{\omega})=0 \quad \text { in } D_{i} \backslash \sum_{j \neq \imath} D_{j} .
$$

Therefore we get

$$
\begin{aligned}
\langle d T, \alpha\rangle & =2 \pi i(-1)^{p} \sum_{i}(-1)^{p-1} \int_{D_{i}} d\left(\operatorname{Res}_{D_{i}}(\omega) \wedge \iota_{D_{i}}^{*} \alpha\right) \\
& =(2 \pi i)^{2} \sum_{i} \sum_{j \neq \imath} \int_{D_{j \cap D_{i}}} \operatorname{Res}_{D_{j} \cdot D_{i}}(\omega) \wedge \iota_{D_{j} \cap D_{i}}^{*} \alpha \\
& =-4 \pi^{2} \sum_{j \neq \imath}\left\langle\operatorname{Res}_{D_{j} \cdot D_{i}}(\omega), \alpha\right\rangle=0 \quad \text { by }(2.3) .
\end{aligned}
$$

Applying Lemma (2.4), we see that

$$
\Theta=\frac{1}{i}(d \Lambda+i \delta) G T
$$


is of type $(p, 0)$ and satisfies $d \Theta=T$. In the same way as in (i) we infer that $S=[\omega]-\Theta$ is harmonic, and so closed. Thus $d \omega=0$ in $M \backslash D$.

\section{REFERENCES}

[D] P. Deligne, Théorie de Hodge, II, Inst. Hautes Études Sci. Publ. Math., 40 (1971), 5-57.

[I] S. IITAKA, On logarithmic Kodaira dimension of algebraic varieties, Complex Analysis and Algebraic Geometry, Ed. W. L. Baily, Jr. and T. Shioda, Iwanami Shoten, Tokyo, 1977, 175-189.

[K] K. KoDAIRA, The theory of harmonic integrals and their applications to algebraic geometry, K. Kodaira: Collected Works Vol. 1, Iwanami Shoten and Princeton Univ. Press, Tokyo-Princeton, 1975, 488-582.

[N1] J. NogUchI, Holomorphic curves in algebraic varieties, Hiroshima Math. J., 7 (1977), 833-853.

[N2] J. Noguchi, Lemma on logarithmic derivatives and holomorphic curves in algebraic varieties, Nagoya Math. J., 83 (1981), 213-233.

[N3] J. NoGuchI, On the value distribution of meromorphic mappings of covering spaces over $\boldsymbol{C}$ into algebraic varieties, J. Math. Soc. Japan, 37 (1985), 295-313.

[N4] J. Noguchi, Logarithmic jet spaces and extensions of de Franchis' theorem, Contributions to Several Complex Variables, Aspects Math. E9, Vieweg, Braunschweig, 1986, 227-249.

[W] A. WEIL, Introduction à l'Étude des Variétés kähleriennes, Hermann, Paris, 1958.

Department of Mathematics

TOKyo Institute of TechNology

Oh-OKayama, Meguro

TOKYO 152 